\title{
Isotopic abundances of Carbon and Oxygen in Oxygen-rich giant stars
}

\author{
Takashi Tsuji ${ }^{1}$ \\ ${ }^{1}$ Institute of Astronomy, The University of Tokyo, Mitaka, Tokyo, 181-0015 Japan \\ email: ttsuji@ioa.s.u-tokyo.ac.jp
}

\begin{abstract}
O} /{ }^{17} \mathrm{O}$ and ${ }^{12} \mathrm{C} /{ }^{13} \mathrm{C}$ ratios in $23 \mathrm{M}$ giants are determined from high resolution IR spectra. The results are confronted with the current models on the convective mixing.
\end{abstract}

Keywords. Convection, stars: abundances, stars: AGB and post-AGB

\section{Introduction}

It has been known that $\mathrm{CNO}$ and their isotopic abundances are useful probes of the mixing in evolved stars. It is, however, by no means clear yet if the observed results could be consistent with the predictions of the stellar evolution models. Here, we concentrate on oxygen-rich giants, possibly experienced the first and second dredges-up, but may not be disturbed by the further processes such as the third dredge-up. For this purpose, we extend our previous analyses on $\mathrm{CO}$ spectra in M giant stars to including isotopic ratios, and we also hope to reexamine the carbon abundances.

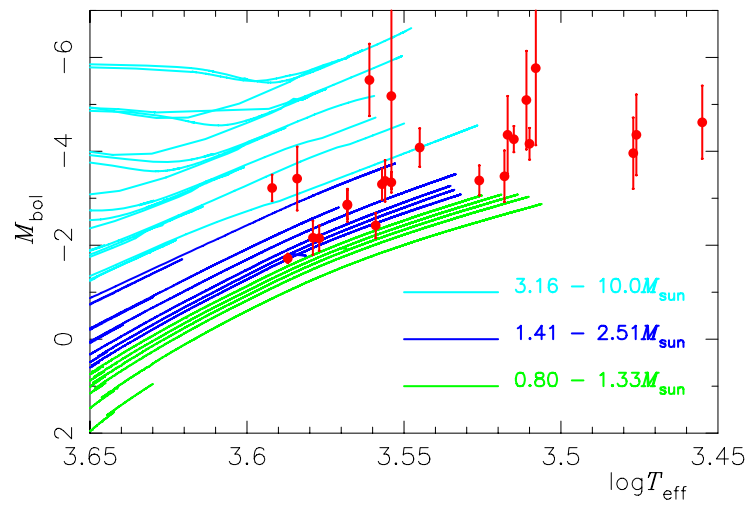

Figure 1. Our sample on the HR diagram and the evolutionary tracks by Claret (2004).

\section{The basic stellar parameters}

We selected 23 red giant stars, for which the effective temperatures are determined by the use of the infrared flux method and the bolometric luminosities by the integration of the SEDs. With the Hipparcos parallaxes, absolute bolometric magnitudes are determined. The error bars of $M_{\mathrm{bol}}$ are based on those of the parallaxes, but errors in photometric data and in $T_{\text {eff }}$ (about $\pm 0.1 \mathrm{mag}$ and $\pm 100 \mathrm{~K}$, respectively) are not included. Our sample is shown on the HR diagram and compared with the evolutionary tracks by Claret (2004) in Fig. 1. Unfortunately, nearly half of our sample cannot be accounted for by the models of Claret, and we had to extrapolate the evolutionary tracks to estimate stellar masses given in Table 1. 


\section{Method of analysis}

We use the high resolution spectra observed with the KPNO FTS, and measured EWs of isolated lines of ${ }^{12} \mathrm{C}^{16} \mathrm{O},{ }^{13} \mathrm{C}^{16} \mathrm{O}$, and ${ }^{12} \mathrm{C}^{17} \mathrm{O}$ in the $K$-band region. We applied a line-by-line analysis on these EWs with the use of the spherically extended $1 \mathrm{D}$ model photospheres. In our analysis, the micro-turbulent velocity $\xi_{\text {mic }}$ is determined so that the abundances of ${ }^{12} \mathrm{C}^{16} \mathrm{O}$ from individual lines show the least dependence on the EWs. The resulting $\xi_{\text {mic }}$ is used to determine abundances of ${ }^{13} \mathrm{C}^{16} \mathrm{O}$ and ${ }^{12} \mathrm{C}^{17} \mathrm{O}$ as well. Some examples are shown in Fig. 2.

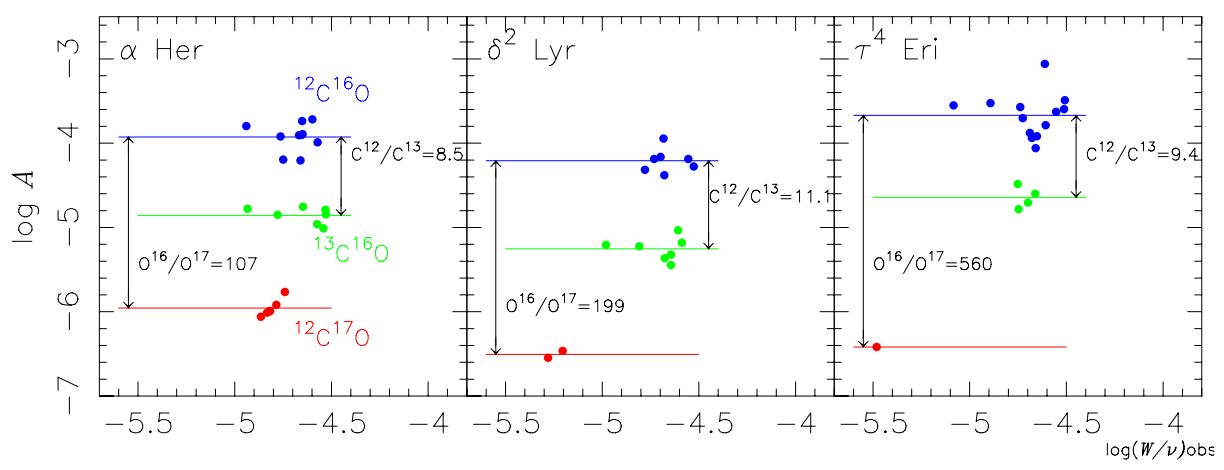

Figure 2. Line-by-line analysis on ${ }^{12} \mathrm{C}^{16} \mathrm{O},{ }^{13} \mathrm{C}^{16} \mathrm{O}$, and ${ }^{12} \mathrm{C}^{17} \mathrm{O}$ in $\alpha$ Her, $\delta^{2}$ Lyr, and $\tau^{4}$ Eri

Table 1. Isotopic Ratios and Basic Stellar Parameters in 23 Red Giant Stars

\begin{tabular}{|c|c|c|c|c|c|c|c|c|c|}
\hline Obj. & $\begin{array}{c}{ }^{12} \mathrm{C} /{ }^{13} \mathrm{C} \\
\text { present }\end{array}$ & $\begin{array}{c}{ }^{12} \mathrm{C} /{ }^{13} \mathrm{C} \\
\text { others }\end{array}$ & $\begin{array}{l}{ }^{16} \mathrm{O} /{ }^{17} \mathrm{O} \\
\text { present }\end{array}$ & $\begin{array}{l}{ }^{16} \mathrm{O} /{ }^{17} \mathrm{O} \\
\text { others }\end{array}$ & $\log A_{\mathrm{C}}$ & $\begin{array}{l}\xi_{\mathrm{mic}} \\
\mathrm{km} \mathrm{s} s^{-1}\end{array}$ & $\begin{array}{c}T_{\text {eff }} \\
\mathrm{K}\end{array}$ & $M_{\mathrm{bol}}$ & $\begin{array}{c}\text { Mass } \\
M_{\odot}\end{array}$ \\
\hline RZ Ari & $5 \pm 1$ & & $402 \pm 57$ & & -4.09 & 2.99 & 3295 & $-3.47 \pm 0.55$ & 1.5 \\
\hline$\alpha$ Cet & $10 \pm 2$ & & 200 & & -3.88 & 3.68 & 3905 & $-3.22 \pm 0.28$ & 3.6 \\
\hline$\rho$ Per & $9 \pm 2$ & $15 \pm 2^{a}$ & 1000 & & -3.76 & 2.86 & 3505 & $-4.08 \pm 0.41$ & 3.2 \\
\hline$\tau^{4}$ Eri & $9 \pm 2$ & & $560 \pm 97$ & & -3.67 & 2.29 & 3700 & $-2.85 \pm 0.35$ & 2.0 \\
\hline$\alpha$ Tau & $11 \pm 2$ & $10 \pm 2^{a}$ & 1000 & $560 \pm 180^{b}$ & -3.71 & 2.59 & 3860 & $-1.72 \pm 0.10$ & 1.4 \\
\hline$\mu \mathrm{Gem}$ & $7 \pm 1$ & $13 \pm 2^{a}$ & $206 \pm 30$ & $325 \pm 112^{b}$ & -4.15 & 3.30 & 3605 & $-3.30 \pm 0.33$ & 2.1 \\
\hline$\nu$ Vir & $7 \pm 1$ & $12 \pm 2^{a}$ & 1500 & & -4.12 & 2.84 & 3795 & $-2.16 \pm 0.36$ & 1.6 \\
\hline$\delta$ Vir & $10 \pm 2$ & $16 \pm 4^{a}$ & 1500 & & -3.91 & 3.33 & 3625 & $-2.42 \pm 0.27$ & 1.4 \\
\hline SW Vir & $11 \pm 2$ & & $188 \pm 22$ & & -4.06 & 4.13 & 2990 & $-4.35 \pm 0.86$ & 1.4 \\
\hline 10 Dra & $11 \pm 2$ & $12 \pm 3^{a}$ & $112 \pm 14$ & & -3.77 & 3.44 & 3700 & $-2.87 \pm 0.31$ & 2.0 \\
\hline RX Boo & $5 \pm 1$ & & $136 \pm 21$ & & -3.75 & 3.08 & 2850 & $-4.62 \pm 0.78$ & 1.4 \\
\hline RR UMi & $5 \pm 1$ & & 1000 & & -4.10 & 3.04 & 3355 & $-3.38 \pm 0.32$ & 1.5 \\
\hline$\sigma \mathrm{Lib}$ & $7 \pm 1$ & & 1500 & & -4.00 & 3.21 & 3600 & $-3.37 \pm 0.44$ & 2.1 \\
\hline$\delta \mathrm{Oph}$ & $9 \pm 1$ & & $232 \pm 46$ & & -3.97 & 3.23 & 3775 & $-2.15 \pm 0.27$ & 1.5 \\
\hline 30g Her & $9 \pm 1$ & $10 \pm 2^{a}$ & $197 \pm 24$ & $675 \pm 175^{c}$ & -4.17 & 3.89 & 3235 & $-4.16 \pm 0.34$ & 1.8 \\
\hline$\alpha$ Her & $9 \pm 1$ & & $107 \pm 14$ & $190 \pm 40^{b}$ & -3.93 & 3.81 & 3220 & $-5.77 \pm 1.64$ & 4.5 \\
\hline OP Her & $9 \pm 1$ & & $184 \pm 21$ & & -4.12 & 4.02 & 3285 & $-4.35 \pm 0.83$ & 2.1 \\
\hline BS6861 & $21 \pm 5$ & & 1000 & & -3.43 & 3.43 & 3580 & $-5.18 \pm 1.98$ & 6.1 \\
\hline XY Lyr & $11 \pm 1$ & & $218 \pm 18$ & & -4.10 & 3.71 & 3245 & $-5.09 \pm 1.05$ & 3.4 \\
\hline$\delta^{2} \mathrm{Lyr}$ & $11 \pm 2$ & & $199 \pm 20$ & & -4.21 & 4.51 & 3637 & $-5.52 \pm 0.77$ & 7.3 \\
\hline R Lyr & $8 \pm 2$ & & $376 \pm 61$ & & -3.73 & 2.76 & 3275 & $-4.26 \pm 0.28$ & 2.0 \\
\hline$\lambda \mathrm{Aqr}$ & $9 \pm 1$ & & 500 & & -3.87 & 3.11 & 3835 & $-3.42 \pm 0.68$ & 3.6 \\
\hline$\beta \mathrm{Peg}$ & $6 \pm 1$ & $8 \pm 2^{a}$ & 1500 & $1050 \pm 375^{b}$ & -4.11 & 3.39 & 3580 & $-3.34 \pm 0.22$ & 2.1 \\
\hline
\end{tabular}

References: a) Smith \& Lambert (1990), b) Harris \& Lambert (1984), c) Harris et al. (1985) 


\section{Results}

The isotopic ratios based on the ${ }^{12} \mathrm{C}^{16} \mathrm{O},{ }^{13} \mathrm{C}^{16} \mathrm{O}$, and ${ }^{12} \mathrm{C}^{17} \mathrm{O}$ abundances are given in Table 1 . The results are further confirmed by a direct comparison of the observed spectra with the synthetic spectra in the regions of the ${ }^{13} \mathrm{C}^{16} \mathrm{O}(2-0)$ bandhead and ${ }^{12} \mathrm{C}^{17} \mathrm{O}(2-0)$ $R 18-33$ lines. Also, we applied the spectral synthesis method to estimate ${ }^{16} \mathrm{O} /{ }^{17} \mathrm{O}$ for the case in which ${ }^{12} \mathrm{C}^{17} \mathrm{O}$ lines are too weak to measure EWs accurately, and the results are given without error bars in Table 1. For a few stars, our results appear to be consistent with the results by other authors (Harris \& Lambert 1984, Harris, Lambert \& Smith 1985, Smith \& Lambert 1990) in general, as shown in Table 1.

\section{${ }^{16} \mathrm{O} /{ }^{17} \mathrm{O}$ Ratio}

The resulting ${ }^{16} \mathrm{O} /{ }^{17} \mathrm{O}$ ratios plotted against the stellar masses are compared with the predicted ones by Claret (2004) (solid lines) in Fig. 3a. The rather large variation of ${ }^{16} \mathrm{O} /{ }^{17} \mathrm{O}$ in low mass stars is well consistent with the prediction of the evolutionary models, confirming the previous analysis by Dearborn (1992) using the observed data known at that time. In the higher mass stars, however, some objects have quite low ${ }^{16} \mathrm{O} /{ }^{17} \mathrm{O}$ ratios and cannot be consistent with the predicted trend.
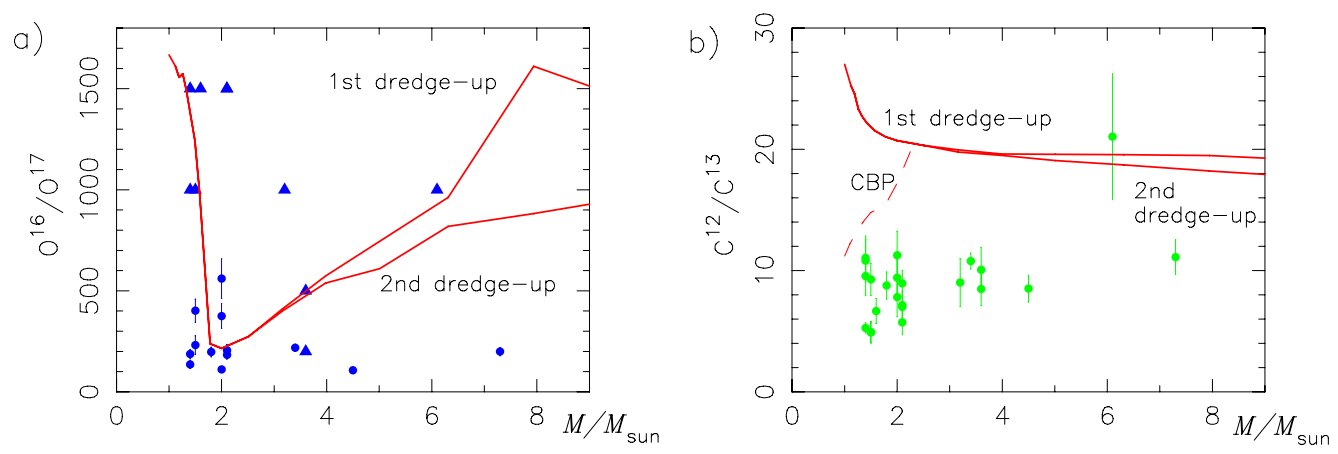

Figure 3. a) Observed and predicted ${ }^{16} \mathrm{O} /{ }^{17} \mathrm{O}$ ratios. b) The same for ${ }^{12} \mathrm{C} /{ }^{13} \mathrm{C}$ ratios

\section{${ }^{12} \mathbf{C} /{ }^{13} \mathbf{C}$ Ratio}

We confirm in Fig. $3 \mathrm{~b}$ that ${ }^{12} \mathrm{C} /{ }^{13} \mathrm{C}$ ratios are mostly around 10 . The contradiction with the evolutionary models, which predict ${ }^{12} \mathrm{C} /{ }^{13} \mathrm{C} \approx 20$ (solid lines), has been a longstanding puzzle. This dilemma may be resolved by assuming a deep circulation below the bottom of the convective zone, referred to as cool bottom processing (Boothroyd \& Sackmann 1999), and its prediction is also plotted in Fig. 3b (dashed line). Our result, however, suggests that the extra mixing may not resolve yet the ${ }^{12} \mathrm{C} /{ }^{13} \mathrm{C}$ puzzle, even if we make allowance for uncertainties in stellar masses.

\section{Concluding remarks}

We also planned to redetermine $\mathrm{C}$ abundances, which showed somewhat different results from the CO 1st (Tsuji 1986) and 2nd (Tsuji 1991) overtone bands, and details on this issue will be discussed elsewhere. Despite such a problem, the isotopic ratios can be determined more accurately than the elemental abundances. For this reason, isotopic ratios can be useful probes of evolutionary models and mixing processes in cool evolved stars. The observed and predicted isotopic ratios, however, are still by no means consistent (Fig. 3). Introduction of an extra-mixing may be needed, but it is vital to pursue a better theory of convection that may comprehend more efficient mixing. 


\section{References}

Boothroyd, A. \& Sackmann, I.-J. 1999, ApJ 510, 232

Claret, A. 2004, A\&SA 424, 919

Dearborn, D. S. P. 1992, Phys. Rep. 210, 367

Harris, M. \& Lambert, D. L. 1984 ApJ 285, 674

Harris, M., Lambert, D. L., \& Smith, V. V. 1985, ApJ 299, 375

Smith, V. V. \& Lambert, D. L. 1990, ApJS 72, 387

Tsuji, T. $1986, A छ A 156,8$

Tsuji, T. 1991, $A \& A 245,203$ 